\title{
Circulating tumor cells in urological cancers
}

\author{
Martin Cegan ${ }^{1}$, Christopher Kobierzycki ${ }^{2}$, Katarina Kolostova ${ }^{3}$, Imrich Kiss ${ }^{3}$, \\ Vladimir Bobek ${ }^{2-4}$, Robert Grill ${ }^{5}$
}

${ }^{1}$ Department of Pathology, Masaryk's Hospital in Usti nad Labem, Usti nad Labem, Czech Republic ${ }^{2}$ Department of Histology and Embryology, Wroclaw Medical University, Wroclaw, Poland

${ }^{3}$ Department of Laboratory Genetics, University Hospital Kralovske Vinohrady, Prague, Czech Republic

${ }^{4}$ Department of Surgery University Hospital Motol and $1{ }^{\text {st }}$ Faculty of Medicine Charles University, Prague, Czech Republic

${ }^{5}$ Department of Urology, University Hospital Kralovske Vinohrady, Prague, Czech Republic

\begin{abstract}
Circulating tumor cells (CTC) represent a very small subpopulation of the cancer cells found in the bloodstream of patients in the metastatic phase of neoplastic disease. Due to the timeline of the disease, they are regarded as a negative prognostic marker. This study focused on determining CTC percentages; these values vary between different types of cancer. In addition to their diagnostic use, CTCs may also be used to treat the disease. Calculating CTC population size and analyzing their biology in patients in advanced stages of cancer may prove valuable in creating a molecular profile for the disease. This would strongly encourage diagnostics and enable personalized treatment. We here present an analysis of recent data on CTCs in urological cancers and their potential uses. (Folia Histochemica et Cytobiologica 2017, Vol. 55, No. 3, 107-113)
\end{abstract}

Key words: CTCs; circulating tumor cells; prostate cancer; kidney cancer; bladder cancer; cultivation; in vitro; gene expression

\section{Introduction}

Disseminated cancer cells that are shed from the primary lesion or from metastatic foci into the peripheral blood are referred to as circulating tumor cells (CTCs). They are mostly found in patients in advanced metastatic stages of the disease, though very rarely they are also observed in premetastatic phases [1, 2]. Nevertheless, their number depends mostly on the phase of the disease and the type and localization of the malignancy. The analysis of their presence and characteristics provides a great amount

\footnotetext{
Correspondence address: V. Bobek, M.D., Ph.D.

University Hospital Kralovske Vinohrady

Department of Laboratory Genetics

Srobarova 50, 10034 Prague, Czech Republic

tel.: +420267163578

e-mail: vbobek@centrum.cz
}

of data that cannot be obtained in any alternate way. First of all, CTCs may indicate the existence of micrometastases, despite negative results in diagnostic imaging. Moreover, the analysis of CTC biology may be used to verify chemoresistance and to evaluate the differences in primary and secondary tumor focus response. Additionally, the presence of CTCs following primary radical treatment suggests the risk of cancer reoccurrence [3]. In view of above outlined features, the application of various methods of CTC testing based on blood filtration may have great clinical impact. There are numerous studies being conducted at any given time to discover reproducible and reliable diagnostic techniques that would enable cells to be obtained for precise analysis and to determine their biological character. The most important goal is to create a reliable and reproducible tool for personalized medicine. The present manuscript presents the state of the art in the use of CTCs as individualized biomarkers in urological cancers. 


\section{Biology of circulating tumor cells}

On account of the many different processes they undergo, the morphology and biology of CTCs is heterogeneous and very complex. Generally, they enter the bloodstream and move to a location characteristic of the tumor type, where they create a metastatic focus. In a dynamically proliferating tumor, formation of blood vessels occurs rapidly. CTCs in these areas find their way into the bloodstream [4]. However, they are detected only very rarely among the billions of other blood cells, even in patients with advanced stages of disease [5]. The use of CTCs in diagnostics seems to be hindered because their presence and number may not correlate with the severity of disease. Besides their scarcity in circulation, CTCs are often highly heterogenic due to metastatic subclone development in the expression of extraordinary mutational profiles during the progression of cancer [6]. In order to cross blood vessels walls, remain invisible to elements of the immune and coagulation systems, and disperse throughout whole body, CTCs must be capable of changing their phenotype [7]. The set of changes that needs to take place is called the epithelial-mesenchymal transition (EMT) [8]. When cells undergoing this transition gain the opportunity to metastasize, they lose the determinants typical of epithelial cells and begin to express antigens characteristic of cells of mesenchymal origin $[9,10]$. First of all, cells undergoing EMT cease or lower the expression of epithelial cell adhesion molecule (EpCAM) and of cytokeratins (CKs). EpCAM is a transmembrane glycoprotein primarily responsible for cell-to-cell adhesion, which is also involved in cell signaling, migration, proliferation, and differentiation. CKs are intracytoplasmic proteins, elements of the cytoskeleton which help epithelial cells resist mechanical stress. Both particles are tissue-specific markers, observed only in cells originating from epithelial tissue $[11,12]$. Simultaneously, the upregulation of mesenchymal markers such as vimentin (VIM), as well as markers of the EMT process, such as twist-related protein (TWIST), is observed. VIM is the major cytoskeletal component of mesenchymal cells, forming intermediate filaments. It plays a significant role in supporting and anchoring the position of organelles in the cytoplasm. TWIST works as a transcription factor, and is one of the hallmarks of EMT. Its activation upregulates $\mathrm{N}$-cadherin and downregulates E-cadherin, as well as induces angiogenesis, extravasation, and chromosomal instability $[11,12]$.

The exceptional heterogeneity of CTCs is clearly visible in advanced breast cancer [13]. Due to their individual cancer-associated marker expression and tumor-seeding potential originating from their phe- notypic characteristics, primary and secondary foci may require different handling. Nowadays, the golden standard in breast cancer diagnostics involves only sampling the primary tumor. In view of the above, CTCs may survive endocrine treatment in patients diagnosed as estrogen receptor (ER)-positive, because CTCs often lack ER expression [13, 14]. In male tumors, decreased therapy effectiveness as a result of CTC presence has also been shown. In prostate cancer, CTCs expressing mutated androgen receptor (AR) and linking variants, such as AR-V7, present antiandrogenic therapy resistance $[15,16]$.

\section{Detection of circulating tumor cells}

There are two main challenges to the detection of CTCs: their rare occurrence in the blood and difficulties in identifying the appropriate cells for further isolation $[15,17]$. To comprehend this extremely small quantity of cells, we need to realize that a $7.5 \mathrm{~mL}$ blood sample obtained from a patient with a solid malignancy contains approximately $1 \mathrm{CTC}$ alongside 10 million white blood cells. Direct identification is based on a combination of negative and positive immunological selection of nonepithelial and epithelial cells, respectively. On the other hand, indirect detection mainly involves the analysis of epithelial-specific mRNA transcripts by RT-PCR. Most existing methods of CTC capture are based on immunoaffinity to EpCAM, which is generally overexpressed in various cancers; it is present in $97 \%$ cases of colon cancers, though only $41.7 \%$ of patients with primary malignant breast cancer show increased EpCAM expression [18, 19]. On the other hand, EpCAM expression appears to be downregulated in CTCs, and the relationship between EpCAM expression at the primary breast cancer focus and in CTCs is unclear [20]. The occurrence of the EMT process with all its features may be a reason for the high rate of false positives in CTC detection based on EpCAM enrichment techniques. The ideal method of CTC detection would be isolation based on cell size, meaning that tumor cells would be captured in accordance with their size from different segments of the peripheral blood. An additional benefit would be the possibility of in vitro cultivation, which would enable differentiation between malignant and benign epithelial cells [21-25].

\section{Use of circulating tumor cells}

The term biomarker encompasses a broad group of determinants, such as clinical, laboratory, genetic, molecular and imaging-based approaches [26]. They are primarily employed in diagnostics to differentiate healthy specimens from pathological specimens, mostly in doubtful cases. They give additional data about 
the advancement of the disease and about survival rates. Moreover, treatment efficacy and recurrence risk may be monitored through marker analysis. The specificity of CTCs as biomarkers is that they represent the individual tumor cells of individual patients and reflect the heterogeneity of possible metastatic sites.

\section{Prostate cancer}

The selection of the most suitable therapy for prostate cancer is currently based on the Gleason score (histopathological analysis) and the serum level of prostate-specific antigen (PSA). Unfortunately, the low specificity of PSA is well known. It can be similarly increased in benign hyperplasia, indolent lesions of epithelial origin (IDLE), and even aggressive lesions [27]. In addition, it is inappropriate for therapy monitoring, due to its low accuracy in treatment response verification.

Nowadays, CTCs are generally accepted as prognostic biomarkers in prostate cancer. The prognostic value of the baseline CTC level has been evaluated in numerous studies in which patients were treated with chemotherapy and androgen receptor (AR) signaling inhibitors [28-33]. Various studies have shown an association between baseline CTCs levels and clinical outcomes in metastatic patients [28, 29, 32, 34]. Decreased levels of CTCs in the bloodstream of patients after therapy correlated with longer overall survival (OS), which was subsequently observed as decreased PSA level and radiographic response [28, 35]. Most importantly, alterations in CTCs levels predate PSA changes, suggesting the usefulness and high accuracy of method for monitoring cancer treatment [29].

Numerous prospective clinical trials have assessed presence of CTCs as an intermediate end point. SWOG S0421 was a phase III double-blind, randomized placebo-controlled trial evaluating patients with metastatic castration-resistant prostate cancer (mCRPC) starting first-line docetaxel chemotherapy with or without atrasentan [30]. Atrasentan is an endothelin receptor antagonist that blocks endothelin-induced cell proliferation. CTCs were found to provide additional discriminatory value (independent prognostic marker) over the PSA level and other factors. Atrasentan had no influence on OS [30].

COU-AA-301 was a phase III double-blind randomized placebo-controlled trial evaluating whether CellSearch-based CTCs enumeration could be used as a surrogate efficacy-response biomarker of OS [32]. Patients with mCRPC who had previously been treated with docetaxel received abiraterone with prednisone versus prednisone alone. Abiraterone is a steroidal CYP17A1 inhibitor and, by extension, an androgen synthesis inhibitor. Baseline favorable versus unfavorable CTCs counts measured on the CellSearch platform were associated with better OS (26 vs. 13 months, HR = 2.74, $\mathrm{p}=0.001)$. Any increase in CTCs count after one cycle of docetaxel treatment was significantly associated with worse OS, whereas a decrease in CTCs counts pointed to an insignificant trend towards improved OS [36]. These data suggest that the detection of rising CTCs counts during docetaxel chemotherapy may be used for clinical verification of the therapy. Moreover, 1195 men with mCRPC who had previously received docetaxel benefited from abiraterone acetate treatment [37]. Thus, CTCs enumeration could be used as a surrogate efficacy-response biomarker of OS [32].

A study analyzing levels of PSA, LDH (lactate dehydrogenase), and CTCs in patients treated with abiraterone showed interesting relationships. Abiraterone treatment $(\mathrm{HR}=0.70, \mathrm{p}<0.0001)$, baseline LDH concentration $(\mathrm{HR}=2.98, \mathrm{p}<0.0001)$, and CTC count $(\mathrm{HR}=1.19, \mathrm{p}<0.0001)$ were prognostic for survival, while PSA level was not $(\mathrm{HR}=1.04$, $p=0.1797$ ) [38]. A "CTCs biomarker panel", composed of CTCS count and LDH serum activity, categorized subjects as low risk (CTCs count $\leq 4$ cells per $7.5 \mathrm{~mL}$ of blood, any LDH), intermediate risk (CTCs count $\geq 5, \mathrm{LDH} \leq 250 \mathrm{U} / \mathrm{L}$ ), and high risk (CTCs count $\geq 5, \mathrm{LDH}>250 \mathrm{U} / \mathrm{L}$ ). The CTCs biomarker panel discriminated survival time and satisfied the four Prentice criteria for surrogacy [38], unlike the CTCs count or LDH as individual variables [32]. These prospective phase III data from SWOG S0421 and COU-AA-301 trials are encouraging and require validation by ongoing, independent phase III clinical trials.

The majority of mCRPC patients are diagnosed in the bone involvement phase. In this case, the Response Evaluation Criteria in Solid Tumors cannot be applied for interpretation. New treatment response biomarkers are thus acutely needed for CRPC patients. The Prostate Cancer Working Group 2 criteria rely on bone scintigraphy and changes in the PSA levels to evaluate response to treatment in these patients [39]. However, progression according to bone scintigraphy is not evaluable before 16 weeks of treatment, and most studies evaluating decrease in PSA levels as a surrogate of survival have yielded negative results; treatment based solely according to PSA values is not recommended [39-42].

Verification of radiological progression free survival (PFS) cannot currently be acquired before at least 12-16 weeks of treatment, and is difficult in the evaluation of widespread bone involvement [43]. An additional post hoc analysis of data for patients 
in prospective IMMC-38 (chemotherapy) and COU-AA-301 (abiraterone) trials with baseline CTCs $\geq 5$ cells per $7.5 \mathrm{~mL}$ was performed in 2016, and evaluated the value of a $30 \%$ CTCs decline from baseline at 4, 8, and 12 weeks of treatment [33]. The $\mathrm{OS}$ in patients with mCRCP after abiraterone and chemotherapy is associated with a $30 \%$ decrease in CTC level from the original number of $\geq 5$ cells per $7.5 \mathrm{~mL}$. Further prospective studies are needed to evaluate this promising surrogate.

\section{Urothelial cancer}

There is still lack of diagnostic biomarkers with satisfactory prognostic and predictive value in urothelial cancers. Besides, all the tests analyze urine samples, and so cannot be used in extravesical tumor diagnostics. The greatest advantages of serum markers are their minimal invasiveness and their possible use in the monitoring of treatment effectiveness, mostly in comparison to surgical biopsies of metastatic sites. There are studies outlining the use of CTCs in superficial and invasive urothelial cancers [44, 45].

It has been shown that CTC-positive patients who have undergone radical cystectomy had a higher risk of recurrence and reduced OS [46, 47]. Moreover, CTC positivity was found to be an isolated risk factor of reduced OS [47, 48]. Alarmingly, the presence of a single CTC in a patient's bloodstream was correlated with reduced survival in patients with urothelial carcinoma of the bladder [46]. Apart from research that examined the quantity of CTCs, there are also studies on their quality. Osman et al. compared CTCs with and without uroplakin/EGFR mRNAs in patients who had undergone radical cystectomy [49]. The first group (uroplakin/EGFR + ) had a higher risk of recurrence. Gudemann et al. analyzed the presence of CKs in CTCs, revealing cancer development potential in CTCs that expressed CK20, which was also associated with clinical stage/disease burden [50].

\section{Renal cancer}

A small number of studies have been published on the detection of CTCs in metastatic renal cell carcinoma (mRCC). An association was found between the presence of CTCs, lymph node involvement, and the presence of metastases at the time of diagnosis in mRCC [51]. Studies have also shown that the presence and quantity of mesenchymal and stem-cell-like CTCs is associated with poor treatment response. The presence of stem-cell-like CD133 + cells or mesenchymal $\mathrm{N}$-cadherin+/CK- cells correlates with shortened PFS.
Moreover, in cases with detectable CD133+ cells, $\mathrm{N}$-cadherin +/CK- cells were often found [52].

\section{Molecular characterization of circulating tumor cells}

The ability to detect CTCs in patients with various diseases has given clinicians the opportunity to improve the diagnostic screening and monitoring of the therapy employed. Moreover, to determine the full informative potential of CTCs, the laboratory tests include DNA, RNA, and protein analysis. Pooled CTCs analyses offer the potential to assess the dominant circulatory clone at a given point in time in a specific patient, and allow the tracking of clonal selections during systemic therapies. The evolution of the molecular development of circulating cells from the primary or metastatic sites could be reproduced by personalized CTCs profiling, which could lead to the ability to detect uncommon resistant clones. For example, AR has recently been a deeply studied area in prostate cancer, also in view of CTCs. The level of expression of complete AR or its splice variants correlates directly with clinical outcome and response $[16,53]$. It has been shown that patients with the expression of AR-V7 (the most frequent variant) present abiraterone and enzalutamide resistance [16]. The clinical importance of circulating AR-V7 as a marker of resistance to inhibition of the androgen/ /androgen receptor axis has been supported by wholeblood PCR assays detecting $A R-V 7$ mRNA without prior CTC enrichment [54]. Similarly, Anonarakis et al. studied patients with overexpressed AR-V7 who were resistant to abiraterone treatment, showing no influence on PSA level [16]. The detection of AR-V7 in $\mathrm{mCRPC}$ patients may lead to a reduction in ineffective abiraterone and enzalutamide treatment, which would be healthful for patients and cost-beneficial to healthcare providers. AR-V7 testing in clinical phase is presently ongoing [54]. However, Bernemann et al. have indicated that patients with $\mathrm{MCRPC}$ and positive AR-V7 CTCs should not be excluded from either next generation androgen deprivation therapy or abiraterone or enzalutamide treatment as long as no appropriate alternative therapy has been introduced [55].

In prostate cancer patients, examination of CTCs revealed mutations in the erythroblastosis virus 26 oncogene homolog gene (ERG). This leads to the synthesis of an ERG oncogene with the AR-driven TMPRSS 2 promoter and has been identified in more than $50 \%$ of hormone-sensitive prostate cancers with preserved further tumor progression [56, 57]. Recognizing this as a potential predictive factor, patients 
with specific ERG mutations can be expected to be more sensitive to abiraterone therapy [58].

The routinely preformed analysis of proliferation marker Ki67 seems to also have diagnostic potential in prostate cancer. Its expression in CTCs positively correlates with expression levels and nuclear localization of ARs, and likewise with the stage of advancement of prostate cancer [59]. Patients with positive Ki-67 CTCs during therapy are theoretically resistant to treatment, and therefore $\mathrm{Ki}-67$ has the potential to be used as marker of therapy effectiveness.

\section{Conclusion}

In summary, it is essential to undertake larger and more detailed analyses of the molecular characteristics of CTCs in urological tumors. Understanding the biology of CTCs will provide a new quality of diagnostic and therapeutic strategies, which will enable effective personalized treatment and prevention of metastatic process.

\section{References}

1. Allard WJ, Matera J, Miller MC, et al. Tumor cells circulate in the peripheral blood of all major carcinomas but not in healthy subjects or patients with nonmalignant diseases. Clin Cancer Res. 2004; 10(20): 6897-6904, doi: 10.1158/1078-0432. CCR-04-0378, indexed in Pubmed: 15501967.

2. Tanaka F, Yoneda K, Kondo N, et al. Circulating tumor cell as a diagnostic marker in primary lung cancer. Clin Cancer Res. 2009; 15(22): 6980-6986, doi: 10.1158/1078-0432.CCR09-1095, indexed in Pubmed: 19887487.

3. Hofman V, Bonnetaud C, Ilie MI, et al. Preoperative circulating tumor cell detection using the isolation by size of epithelial tumor cell method for patients with lung cancer is a new prognostic biomarker. Clin Cancer Res. 2011; 17(4): 827-835, doi: 10.1158/1078-0432.CCR-10-0445, indexed in Pubmed: 21098695.

4. Liberko M, Kolostova K, Bobek V. Essentials of circulating tumor cells for clinical research and practice. Crit Rev Oncol Hematol. 2013; 88(2): 338-356, doi: 10.1016/j.critrevonc.2013.05.002, indexed in Pubmed: 23830807.

5. Yu M, Stott S, Toner M, et al. Circulating tumor cells: approaches to isolation and characterization. J Cell Biol. 2011; 192(3): 373-382, doi: 10.1083/jcb.201010021, indexed in Pubmed: 21300848 .

6. Turajlic S, Swanton C. Metastasis as an evolutionary process. Science. 2016; 352(6282): 169-175, doi: 10.1126/science. aaf2784, indexed in Pubmed: 27124450.

7. Pantel K, Brakenhoff RH. Dissecting the metastatic cascade. Nat Rev Cancer. 2004; 4(6): 448-456, doi: 10.1038/nrc1370, indexed in Pubmed: 15170447.

8. Książkiewicz M, Markiewicz A, Zaczek AJ. Epithelial-mesenchymal transition: a hallmark in metastasis formation linking circulating tumor cells and cancer stem cells. Pathobiology. 2012; 79(4): 195-208, doi: 10.1159/000337106, indexed in Pubmed: 22488297.

9. Guarino M. Epithelial-mesenchymal transition and tumour invasion. Int J Biochem Cell Biol. 2007; 39(12): 2153-2160, doi: 10.1016/j.biocel.2007.07.011, indexed in Pubmed: 17825600.
10. Zeisberg M, Neilson EG. Biomarkers for epithelial-mesenchymal transitions. J Clin Invest. 2009; 119(6): 1429-1437, doi: 10.1172/JCI36183, indexed in Pubmed: 19487819.

11. Kalluri R. EMT: when epithelial cells decide to become mesenchymal-like cells. J Clin Invest. 2009; 119(6): 1417-1419, doi: 10.1172/JCI39675, indexed in Pubmed: 19487817.

12. Thiery JP. Epithelial-mesenchymal transitions in tumour progression. Nat Rev Cancer. 2002; 2(6): 442-454, doi: 10.1038/ nrc822, indexed in Pubmed: 12189386.

13. Babayan A, Hannemann J, Spötter J, et al. Heterogeneity of estrogen receptor expression in circulating tumor cells from metastatic breast cancer patients. PLoS One. 2013; 8(9): e75038, doi: 10.1371/journal.pone.0075038, indexed in Pubmed: 24058649.

14. Paoletti C, Larios JM, Muniz MC, et al. Heterogeneous estrogen receptor expression in circulating tumor cells suggests diverse mechanisms of fulvestrant resistance. Mol Oncol. 2016; 10(7): 1078-1085, doi: 10.1016/j.molonc.2016.04.006, indexed in Pubmed: 27178224.

15. Miyamoto DT, Zheng Yu, Wittner BS, et al. RNA-Seq of single prostate CTCs implicates noncanonical Wnt signaling in antiandrogen resistance. Science. 2015; 349(6254): 1351-1356, doi: 10.1126/science.aab0917, indexed in Pubmed: 26383955.

16. Antonarakis ES, Lu C, Wang $\mathrm{H}$, et al. AR-V7 and resistance to enzalutamide and abiraterone in prostate cancer. N Engl J Med. 2014; 371(11): 1028-1038, doi: 10.1056/NEJMoa1315815, indexed in Pubmed: 25184630.

17. Menen RS, Pinney E, Kolostova K, et al. A rapid imageable in vivo metastasis assay for circulating tumor cells. Anticancer Res. 2011; 31(10): 3125-3128, indexed in Pubmed: 21965717.

18. Spizzo G, Went P, Dirnhofer S, et al. High Ep-CAM expression is associated with poor prognosis in node-positive breast cancer. Breast Cancer Res Treat. 2004; 86(3): 207-213, doi: 10.1023/B: BREA.0000036787.59816.01, indexed in Pubmed: 15567937.

19. Spizzo G, Fong D, Wurm M, et al. EpCAM expression in primary tumour tissues and metastases: an immunohistochemical analysis. J Clin Pathol. 2011; 64(5): 415-420, doi: 10.1136/ jcp.2011.090274, indexed in Pubmed: 21415054.

20. Rao CG, Chianese D, Doyle GV, et al. Expression of epithelial cell adhesion molecule in carcinoma cells present in blood and primary and metastatic tumors. Int J Oncol. 2005; 27(1): 49-57, indexed in Pubmed: 15942643.

21. Bobek V, Cegan M, Kolostova K. Circulating tumour cells in patients with urothelial tumours: Enrichment and in vitro culture. Can Urol Assoc J. 2014; 8(9-10): E715-E720, doi: 10.5489/cuaj.1978, indexed in Pubmed: 25408812.

22. Menen R, Pinney E, Hassanein MK, et al. Inhibition of metastasis of circulating human prostate cancer cells in the chick embryo by an extracellular matrix produced by foreskin fibroblasts in culture. Anticancer Res. 2012; 32(5): 1573-1577, indexed in Pubmed: 22593434.

23. Bobek V, Hoffman RM, Kolostova K. Site-specific cytomorphology of disseminated PC-3 prostate cancer cells visualized in vivo with fluorescent proteins. Diagn Cytopathol. 2013; 41(5): 413-417, doi: 10.1002/dc.22843, indexed in Pubmed: 22508666.

24. Kolostova K, Broul M, Schraml J, et al. Circulating tumor cells in localized prostate cancer: isolation, cultivation in vitro and relationship to T-stage and Gleason score. Anticancer Res. 2014; 34(7): 3641-3646, indexed in Pubmed: 24982381.

25. Kolostova K, Pinterova D, Hoffman RM, et al. Circulating human prostate cancer cells from an orthotopic mouse model rapidly captured by immunomagnetic beads and imaged 
by GFP expression. Anticancer Res. 2011; 31(5): 1535-1539, indexed in Pubmed: 21617207.

26. Wagner JA, Williams SA, Webster CJ. Biomarkers and surrogate end points for fit-for-purpose development and regulatory evaluation of new drugs. Clin Pharmacol Ther. 2007; 81(1): 104-107, doi: 10.1038/sj.clpt.6100017, indexed in Pubmed: 17186007.

27. Humphrey PA, Moch H, Cubilla AL, et al. The 2016 WHO Classification of Tumours of the Urinary System and Male Genital Organs-Part B: Prostate and Bladder Tumours. Eur Urol. 2016; 70(1): 106-119, doi: 10.1016/j.eururo.2016.02.028, indexed in Pubmed: 26996659.

28. de Bono JS, Kristeleit R, Tolcher A, et al. Phase I pharmacokinetic and pharmacodynamic study of LAQ824, a hydroxamate histone deacetylase inhibitor with a heat shock protein-90 inhibitory profile, in patients with advanced solid tumors. Clin Cancer Res. 2008; 14(20): 6663-6673, doi: 10.1158/1078-0432. CCR-08-0376, indexed in Pubmed: 18927309.

29. Scher HI, Jia X, de Bono JS, et al. Circulating tumour cells as prognostic markers in progressive, castration-resistant prostate cancer: a reanalysis of IMMC38 trial data. Lancet Oncol. 2009; 10(3): 233-239, doi: 10.1016/S1470-2045(08)70340-1, indexed in Pubmed: 19213602.

30. Goldkorn A, Ely B, Quinn DI, et al. Circulating tumor cell counts are prognostic of overall survival in SWOG S0421: a phase III trial of docetaxel with or without atrasentan for metastatic castration-resistant prostate cancer. J Clin Oncol. 2014; 32(11): 1136-1142, doi: 10.1200/JCO.2013.51.7417, indexed in Pubmed: 24616308

31. Fleisher M, Danila DC, Fizazi K, et al. Circulating tumor cell (CTC) enumeration in men with metastatic castration-resistant prostate cancer (mCRPC) treated with enzalutamide post-chemotherapy (phase 3 AFFIRM study). J Clin Oncol. 2015; 33(Suppl): 5035.

32. Scher HI, Heller G, Molina A, et al. Circulating tumor cell biomarker panel as an individual-level surrogate for survival in metastatic castration-resistant prostate cancer. J Clin Oncol. 2015; 33(12): 1348-1355, doi: 10.1200/JCO.2014.55.3487, indexed in Pubmed: 25800753.

33. Lorente D, Olmos D, Mateo J, et al. Decline in Circulating Tumor Cell Count and Treatment Outcome in Advanced Prostate Cancer. Eur Urol. 2016; 70(6): 985-992, doi: 10.1016/j. eururo.2016.05.023, indexed in Pubmed: 27289566.

34. Danila DC, Heller G, Gignac GA, et al. Circulating tumor cell number and prognosis in progressive castration-resistant prostate cancer. Clin Cancer Res. 2007; 13(23): 7053-7058, doi: 10.1158/1078-0432.CCR-07-1506, indexed in Pubmed: 18056182.

35. Sonpavde G, Pond GR, Berry WR, et al. The association between radiographic response and overall survival in men with metastatic castration-resistant prostate cancer receiving chemotherapy. Cancer. 2011; 117(17): 3963-3971, doi: 10.1002/cncr.25982, indexed in Pubmed: 21365623.

36. Quinn DI, Tangen CM, Hussain M, et al. Docetaxel and atrasentan versus docetaxel and placebo for men with advanced castration-resistant prostate cancer (SWOG S0421): a randomised phase 3 trial. Lancet Oncol. 2013; 14(9): 893-900, doi: 10.1016/S1470-2045(13)70294-8, indexed in Pubmed: 23871417.

37. de Bono JS, Logothetis CJ, Molina A, et al. COU-AA-301 Investigators. Abiraterone and increased survival in metastatic prostate cancer. N Engl J Med. 2011; 364(21): 1995-2005, doi: 10.1056/NEJMoa1014618, indexed in Pubmed: 21612468.

38. Prentice RL. Surrogate endpoints in clinical trials: definition and operational criteria. Stat Med. 1989; 8(4): 431-440, indexed in Pubmed: 2727467.
39. Scher HI, Halabi S, Tannock I, et al. Prostate Cancer Clinical Trials Working Group. Design and end points of clinical trials for patients with progressive prostate cancer and castrate levels of testosterone: recommendations of the Prostate Cancer Clinical Trials Working Group. J Clin Oncol. 2008; 26(7): 1148-1159, doi: 10.1200/JCO.2007.12.4487, indexed in Pubmed: 18309951.

40. Halabi S, Armstrong AJ, Sartor O, et al. Prostate-specific antigen changes as surrogate for overall survival in men with metastatic castration-resistant prostate cancer treated with second-line chemotherapy. J Clin Oncol. 2013; 31(31): 3944-3950, doi: 10.1200/JCO.2013.50.3201, indexed in Pubmed: 24101043.

41. Armstrong AJ, Garrett-Mayer E, Ou Yang YC, et al. Prostate-specific antigen and pain surrogacy analysis in metastatic hormone-refractory prostate cancer. J Clin Oncol. 2007; 25(25): 3965-3970, doi: 10.1200/JCO.2007.11.4769, indexed in Pubmed: 17761981.

42. Petrylak DP, Ankerst DP, Jiang CS, et al. Evaluation of prostate-specific antigen declines for surrogacy in patients treated on SWOG 99-16. J Natl Cancer Inst. 2006; 98(8): 516-521, doi: 10.1093/jnci/dji129, indexed in Pubmed: 16622120.

43. Morris MJ, Molina A, Small EJ, et al. Radiographic progression-free survival as a response biomarker in metastatic castration-resistant prostate cancer: COU-AA-302 results. J Clin Oncol. 2015; 33(12): 1356-1363, doi: 10.1200/ JCO.2014.55.3875, indexed in Pubmed: 25624432.

44. Abbosh PH, Rosenberg JE, Plimack ER. Circulating biomarkers to guide systemic therapy for urothelial carcinoma. Urol Oncol. 2016; 34(11): 502-509, doi: 10.1016/j.urolonc.2016.08.019, indexed in Pubmed: 27751785.

45. Cegan M, Kolostova K, Matkowski R, et al. In vitro culturing of viable circulating tumor cells of urinary bladder cancer. Int J Clin Exp Pathol. 2014; 7(10): 7164-7171, indexed in Pubmed: 25400813.

46. Cowan NC, Crew JP. Imaging bladder cancer. Curr Opin Urol. 2010; 20(5): 409-413, doi: 10.1097/MOU.0b013e32833cbcb9, indexed in Pubmed: 20625298.

47. Gradilone A, Petracca A, Nicolazzo C, et al. Prognostic significance of survivin-expressing circulating tumour cells in T1G3 bladder cancer. BJU Int. 2010; 106(5): 710-715, doi: 10.1111/j.1464-410X.2009.09130.x, indexed in Pubmed: 20128783.

48. Rink M, Chun FKH, Minner S, et al. Detection of circulating tumour cells in peripheral blood of patients with advanced non-metastatic bladder cancer. BJU Int. 2011; 107(10): 1668-1675, doi: 10.1111/j.1464-410X.2010.09562.x, indexed in Pubmed: 20735381.

49. Osman I, Kang M, Lee A, et al. Detection of circulating cancer cells expressing uroplakins and epidermal growth factor receptor in bladder cancer patients. Int J Cancer. 2004; 111(6): 934-939, doi: 10.1002/ijc.20366, indexed in Pubmed: 15300806.

50. Güdemann CJ, Weitz J, Kienle P, et al. Detection of hematogenous micrometastasis in patients with transitional cell carcinoma. J Urol. 2000; 164(2): 532-536, indexed in Pubmed: 10893639.

51. Bluemke K, Bilkenroth U, Meye A, et al. Detection of circulating tumor cells in peripheral blood of patients with renal cell carcinoma correlates with prognosis. Cancer Epidemiol Biomarkers Prev. 2009; 18(8): 2190-2194, doi: 10.1158/10559965.EPI-08-1178, indexed in Pubmed: 19661076.

52. Nel I, Gauler TC, Bublitz K, et al. Circulating Tumor Cell Composition in Renal Cell Carcinoma. PLoS One. 2016; 11(4): e0153018, doi: 10.1371/journal.pone.0153018, indexed in Pubmed: 27101285. 
53. Miyamoto DT, Lee RJ, Stott SL, et al. Androgen receptor signaling in circulating tumor cells as a marker of hormonally responsive prostate cancer. Cancer Discov. 2012; 2(11): 995-1003, doi: 10.1158/2159-8290.CD-12-0222, indexed in Pubmed: 23093251.

54. Markowski MC, Frick KD, Eshleman JR, et al. Cost-savings analysis of AR-V7 testing in patients with metastatic castration-resistant prostate cancer eligible for treatment with abiraterone or enzalutamide. Prostate. 2016; 76(16): 1484-1490, doi: 10.1002/pros.23232, indexed in Pubmed: 27404039.

55. Bernemann C, Schnoeller TJ, Luedeke M, et al. Expression of AR-V7 in circulating tumour cells does not preclude response to next generation androgen deprivation therapy in patients with castration resistant prostate cancer. Eur Urol. 2017; 71(1): 1-3, doi: 10.1016/j.eururo.2016.07.021, indexed in Pubmed: 27471164.

56. Robinson D, Van Allen EM, Wu YM, et al. Integrative clinical genomics of advanced prostate cancer. Cell. 2015;
161(5): 1215-1228, doi: 10.1016/j.cell.2015.05.001, indexed in Pubmed: 26000489.

57. Barbieri CE, Bangma $\mathrm{CH}$, Bjartell $\mathrm{A}$, et al. The mutational landscape of prostate cancer. Eur Urol. 2013; 64(4): 567-576, doi: 10.1016/j.eururo.2013.05.029, indexed in Pubmed: 23759327.

58. Attard G, de Bono JS, Logothetis CJ, et al. Improvements in radiographic progression-free survival stratified by ERG gene status in metastatic castration-resistant prostate cancer patients treated with abiraterone acetate. Clin Cancer Res. 2015; 21(7): 1621-1627, doi: 10.1158/1078-0432.CCR-14-1961, indexed in Pubmed: 25593303.

59. Reyes EE, VanderWeele DJ, Isikbay M, et al. Quantitative characterization of androgen receptor protein expression and cellular localization in circulating tumor cells from patients with metastatic castration-resistant prostate cancer. J Transl Med. 2014; 12: 313, doi: 10.1186/s12967-014-0313-z, indexed in Pubmed: 25424879.

Submitted: 28 December, 2016 Accepted after reviews: 18 September, 2017 Available as AoP: 29 September, 2017 